\title{
Clinical Outcome in Male Patients With Detrusor Overactivity With Impaired Contractility
}

\author{
Shuo Liu, Lewis Chan, Vincent Tse \\ Department of Urology, Concord Repatriation General Hospital, Sydney, Australia
}

\begin{abstract}
Purpose: To review the clinical outcomes of patients with voiding dysfunction who have detrusor overactivity with impaired contractility (DOIC) diagnosed with urodynamic studies.

Methods: Urodynamic reports from 2005 to 2009 were reviewed, and 54 male patients had findings consistent with DOIC. Patients with acontractile or neuropathic bladders were excluded. Clinical outcomes were obtained from patient records.

Results: Of 54 men, 8 presented with voiding symptoms, 17 had storage symptoms, and 29 had mixed symptoms. Twenty-two had a previous transurethral resection of the prostate. The median follow-up was 12 months. Four patients received no intervention. Two patients were taught intermittent self-catheterization. Five patients underwent surgery to reduce outlet resistance and all reported improvement. Forty-three patients were started on pharmacotherapy; symptomatic improvement was reported by 9 of 16 patients commenced on anticholinergics alone, 6 of 16 on alpha-blockers alone, and 4 of 5 treated with a combination of alpha-blockers and anticholinergics. Eleven patients experienced no difference on pharmacotherapy and 2 reported deterioration. One patient developed acute urinary retention (18 months after commencing treatment with alpha-blockers). No patient had urosepsis.

Conclusions: Anticholinergics and alpha-blockers appear to be safe in patients with DOIC. The risk of urinary retention and sepsis is low. The majority of patients report symptomatic benefit from either drugs or surgical treatment.
\end{abstract}

Keywords: Lower urinary tract symptoms; Overactive urinary bladder; Urodynamics; Cholinergic antagonists; Adrenergic alpha-antagonists

\section{INTRODUCTION}

Detrusor overactivity with impaired contractility (DOIC), traditionally referred to as detrusor hyperactivity with impaired contractility (DHIC), was first characterized by Resnick and Yalla [1] in 1987 as a type of voiding dysfunction in the elderly [2]. There has been much ongoing debate regarding its underlying etiology and whether it is caused by a single pathophysiological mechanism or merely represents the coexistence of two very commonly seen but independent conditions, detrusor overactivity and hypocontractility [3-6]. Although initially described in frail institutionalized elderly, DOIC has been increasingly recognized as a cause of lower urinary tract symptoms (LUTS) in community dwelling men [7-9]. In a recent study, Abarbanel and Mar- cus [9] showed that DOIC was the principal dysfunction in up to $18 \%$ of elderly patients referred for urodynamic studies and was more commonly seen in men. Our previous urodynamic study in elderly women demonstrated that the condition occurred in $8 \%$ of our cohort of octogenarians [10].

Clinically, DOIC is often mistaken for other common pathologies such as benign prostatic hyperplasia (BPH) in men and stress incontinence in women. It is an urodynamic diagnosis and is characterized by involuntary detrusor contractions or reduced compliance on filling and a low-pressure low-flow voiding pattern. DOIC presents a unique clinical dilemma in which therapeutic options targeting one component of the disease may potentially aggravate the other. Anticholinergic use has been discouraged in the past for fear of inducing urinary
Corresponding author: Shuo Liu

Department of Urology, Concord Repatriation General Hospital, Hospital Road, Concord West, NSW 2138, Australia

Tel: +61 404865180 / Fax: +61 288192099 / E-mail: shuo.mark.liu@hotmail.com Submitted: June 29, 2014 / Accepted after revision: September 1, 2014
This is an Open Access article distributed under the terms of the Creative Commons Attribution Non-Commercial License (http://creativecommons.org/licenses/by-nc/3.0/) which permits unrestricted non-commercial use, distribution, and reproduction in any medium, provided the original work is properly cited. 
retention in patients with coexisting detrusor failure $[1,11]$. Despite its prevalence in the elderly, there is currently no literature describing the clinical course of DOIC. We report the clinical course of a group of patients with DOIC and postulate that, with correct diagnosis and accurate characterization of this complex dysfunction using urodynamics, patients can benefit from targeted therapy comprised of conventional pharmacotherapy and surgery.

\section{MATERIALS AND METHODS}

Multichannel urodynamic studies, according to the International Continence Society (ICS) standards, were performed on more than 1,500 community-dwelling patients at our outpatient department between June 2005 and November 2009. Male patients with the DOIC diagnosis were identified.

Currently, no official ICS definitions for DOIC and hypocontractility exist. For the purpose of this study, we defined detrusor hyperactivity as either phasic detrusor contraction (detrusor overactivity, DO) and/or reduced compliance (with $\Delta$ Pdet $>15$ $\mathrm{cm} \mathrm{H}_{2} \mathrm{O}$ ) on filling. Hypocontractility was characterized by detrusor contraction of reduced strength and/or duration, resulting in prolonged bladder emptying. A bladder contractility index (BCI) was calculated using the following formula: $\mathrm{BCI}=$ detrusor pressure at maximal flow rate (PdetQmax) +5 maximal flow rate (Qmax). Impaired contractility was represented by a value $<100$ [12]. Patients with acontractile detrusor, long-term indwelling urethral catheters, and neuropathic bladder dysfunction were excluded from our study, as these patients usually follow a different clinical course and are less likely to respond to medical and surgical interventions.

Fifty-four men demonstrated features suggestive of DOIC. For each patient, the assessing urologist completed an urodynamic proforma, which included the reason for referral, symptoms, previous history, and medications. Recorded symptoms included storage symptoms (frequency, urgency, and nocturia) and voiding symptoms (poor stream, hesitancy, and intermittency), as well as any associated incontinence. Management options included pharmacological agents, surgical intervention, and conservative measures. Treatment decisions were based on patient presentation, baseline fitness, and urodynamic parameters. We retrospectively reviewed all clinic letters and patient notes to determine patients' subjective symptom outcomes and any complications related to the treatment prescribed.

Statistical analysis was performed using the SPSS ver. 17.0 (SPSS Inc., Chicago, IL, USA). Clinical characteristics, urodynamic parameters, and clinical outcomes were compared using the chi-square test, Mann-Whitney test, and Student t-test where appropriate. A significant difference was defined as $\mathrm{P}<0.05$.

\section{RESULTS}

Fifty-four men, with a mean age of 76.3 years (range, 51-101 years), had urodynamic findings consistent with DOIC (Table 1). The median follow-up period was 12 months (range, 3-39 months). Eight patients (14.8\%) presented with voiding symptoms, 17 (31.5\%) had storage symptoms, and 29 (53.7\%) had mixed symptoms. At the time of the urodynamic study, 22 men

Table 1. Characteristics of 54 men diagnosed with DOIC, by clinical presentation (voiding, storage, and mixed symptoms)

\begin{tabular}{|c|c|c|c|c|c|}
\hline Variable & Total $(n=54)$ & Voiding $(n=8)$ & Storage $(n=17)$ & Mixed $(n=29)$ & P-value \\
\hline Age (yr) & $76.3 \pm 10.9$ & $83.13 \pm 10.6$ & $77.9 \pm 7.5$ & $73.5 \pm 11.9$ & 0.06 \\
\hline Detrusor overactivity & $18(33.3)$ & 3 & 7 & 8 & 0.62 \\
\hline Reduced compliance & $26(48.1)$ & 4 & 6 & 16 & 0.42 \\
\hline Both features present & $10(18.5)$ & 1 & 4 & 5 & 0.78 \\
\hline Urge incontinence & $7(13.0)$ & 1 & 2 & 4 & 0.98 \\
\hline Cystometric volume (mL) & $366.0 \pm 105.9$ & $364.0 \pm 103.4$ & $378.7 \pm 105.7$ & $358.6 \pm 109.8$ & 0.83 \\
\hline $\mathrm{Qmax}(\mathrm{mL} / \mathrm{sec})$ & $8.3 \pm 4.7$ & $4.7 \pm 3.4$ & $11 \pm 5.7$ & $7.6 \pm 3.5$ & $<0.01$ \\
\hline PdetQmax $\left(\mathrm{cm} \mathrm{H}_{2} \mathrm{O}\right)$ & $31.1 \pm 10$ & $31.7 \pm 13.6$ & $33.5 \pm 8.6$ & $29.4 \pm 10.1$ & 0.44 \\
\hline PVR volume (mL) & $101.8 \pm 112.2$ & $203.8 \pm 169.3$ & $62 \pm 72.8$ & $93.9 \pm 95.4$ & 0.01 \\
\hline Abdominal straining & $17(31.5)$ & 1 & 6 & 10 & 0.45 \\
\hline
\end{tabular}

Values are presented as mean \pm standard deviation.

IPSS, international prostate symptom score; $\mathrm{BPH}$, benign prostatic hyperplasia; PSA, prostate-specific antigen. 


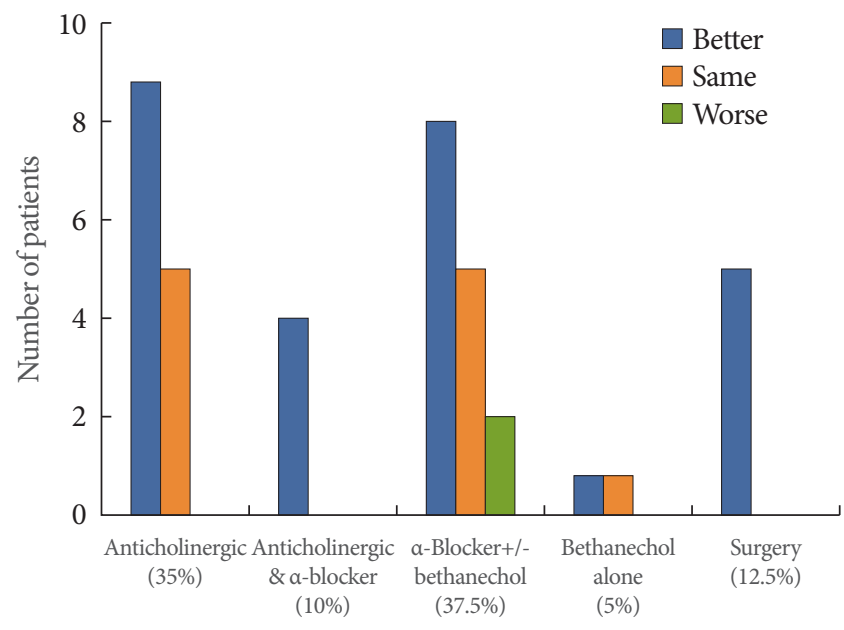

Type of treatment instituted (\% of 40 patients who returned for follow-up)

Fig. 1. Symptomatic response in 40 men after pharmaceutical or surgical intervention (excluding 8 patients did not attend subsequent follow-up).

(40.7\%) had persistent LUTS despite previous surgery aimed at reducing bladder outlet obstruction (BOO; transurethral resection of the prostate [TURP] in 20 and bladder neck incision [BNI] in 2) and 3 had a history of acute urinary retention requiring short-term catheterization.

On filling cystometry, 26 patients (48.1\%) had reduced compliance, 18 (33.3\%) had phasic detrusor contractions, and 10 (18.5\%) demonstrated both features of detrusor overactivity. Seven men experienced leakage secondary to urge incontinence. The mean cystometric capacity was $366.0 \mathrm{~mL}$. During voiding, the mean Qmax was $8.3 \mathrm{~mL} / \mathrm{sec}$, the mean PdetQmax was $31.1 \mathrm{~cm} \mathrm{H}_{2} \mathrm{O}$, and the mean postvoid residual volume (PVR) was $101.8 \mathrm{~mL}$. Seventeen men (31.5\%) displayed signs of abdominal straining; for the remaining patients, the mean BCI was 68. There were no posturodynamic complications.

Four patients received no intervention because they had manageable symptoms, and 2 were taught intermittent selfcatheterization. Forty-three men were started on pharmacotherapy, and 5 underwent surgery to reduce outlet resistance. Twenty-seven of 48 patients (56\%) who had received active treatment (pharmaceutical or surgical) for their LUTS reported symptom improvement on follow-up (Fig. 1). Eight patients (17\%) did not return for follow-up. In the remaining 13 men (27\%), only 2 (4\%) experienced symptom progression while 11 (23\%) noted no significant change.

Table 2 shows a comparison between the 27 men with symptomatic improvement during the follow-up period and the 13
Table 2. Comparison of characteristics of men with and without symptomatic improvement

\begin{tabular}{lccc}
\hline Characteristic & Better $(\mathrm{n}=27)$ & $\begin{array}{c}\text { Same or worse } \\
(\mathrm{n}=13)\end{array}$ & P-value \\
\hline Age $(\mathrm{yr})$ & $78.3 \pm 9.7$ & $77 \pm 11.5$ & 0.72 \\
Presentation & & & 0.94 \\
$\quad$ V/S/M & $4 / 9 / 14$ & $2 / 5 / 6$ & \\
$\begin{array}{l}\text { Both features of DO \& } \\
\text { reduced compliance }\end{array}$ & 7 & 1 & 0.18 \\
Urge incontinence & 6 & 1 & 0.26 \\
Cystometric volume $(\mathrm{mL})$ & $385.8 \pm 120.5$ & $299.3 \pm 81.0$ & 0.26 \\
Qmax (mL/s) & $8.8 \pm 5.6$ & $7.3 \pm 4.3$ & 0.40 \\
PdetQmax (cm $\left.\mathrm{H}_{2} \mathrm{O}\right)$ & $28.1 \pm 10.7$ & $34.2 \pm 7.5$ & 0.90 \\
PVR volume $(\mathrm{mL})$ & $105.2 \pm 102.9$ & $81.5 \pm 108.5$ & 0.65 \\
Abdominal straining & 10 & 2 & 0.16 \\
\hline
\end{tabular}

Values are presented as mean \pm standard deviation.

V, voiding; S, storage; M, mixed; DO, detrusor overactivity; Qmax, maximal flow rate; PdetQmax, detrusor pressure at maximal flow rate; PVR, postvoid residual.

men without improvement. There was no significant difference between the two groups in terms of age, type of presenting LUTS, and urodynamic parameters. Patients with both features of hyperactivity (i.e., reduced compliance as well as phasic contraction) were more likely to perceive improvement, although this was not statistically significant. Similarly, the presence of urge incontinence or abdominal straining during voiding did not correlate with poor symptomatic outcomes after treatment. In fact, the majority of these men had good treatment responses.

In terms of complications, one patient developed acute urinary retention 18 months after beginning treatment with alpha-blockers. There were also 3 cases of uncomplicated urinary tract infection. No patient developed urosepsis during the follow-up period.

\section{DISCUSSION}

DOIC poses several diagnostic and therapeutic challenges. In addition to being a paradoxical dysfunction involving both filling and voiding phases, patients are mostly elderly with various comorbidities [9]. In clinical practice, many patients have been treated empirically (and perhaps incorrectly) based on their symptoms [7].

Multiple studies have repeatedly demonstrated that LUTS alone correlate poorly with the underlying urodynamic diagnosis; this is especially true in the geriatric population $[10,13,14]$. 
The clinical presentation is often a result of the complex interplay between multiple pathophysiological processes: the effect of an aging bladder, the presence of $\mathrm{BOO}$, and systemic factors such as polypharmacy and declining cardiovascular and cognitive functions. In clinical practice, however, most elderly men presenting with LUTS are empirically treated for BPH using alpha-blockers and, in refractory cases, prostatectomy and/or BNI. Dissatisfaction with surgical outcome is as high as 25\% [15]. In a 2002 study, Kuo [7] investigated the cause of LUTS in 185 postprostatectomy patients and found BOO in only 52 men (28\%). The author suggested that incorrect preoperative diagnosis might have been the main reason for subsequent surgical failure.

It is important to remember that prescribed treatments are often not benign. The perioperative morbidity of TURP has been reported at around 20\%, and the risks of anesthesia and surgery increase with age $[16,17]$. Prostatectomy may exacerbate certain components of LUTS, such as urinary incontinence, in individuals with concomitant DO $[7,18,19]$. Side effects of pharmaceutical agents are also more pronounced in the elderly and are associated with substantially higher morbidity and mortality. An example of this is the higher incidence of falls and fall-related hospital admissions observed in elderly patients taking alpha-blockers and anticholinergics [20,21].

The DOIC diagnosis can only be established on urodynamics. A pressure-flow study can provide crucial information on the extent and the type of detrusor dysfunction and the level of functional reserve, allowing for more targeted therapy, even in those with previous surgery. In our study, among the 22 men who had been empirically treated with either TURP or BNI, 18 later underwent conventional pharmacotherapy, and one had revision TURP based on the recommendations of the urodynamic findings. Eleven subsequently improved. More importantly, urodynamic studies alert the treating physician to clinical pitfalls common in DOIC, such as prescribing high-dose anticholinergics to a patient with storage symptoms but minimal detrusor contractility. At present, the routine use of urodynamic studies in the assessment of LUTS is not backed by most official guidelines [22]. However, we believe that it has a potentially vital role in the management of those who are vulnerable to treatment side effects and whose underlying etiology is likely to be complex. It should be considered in elderly patients who are otherwise ambulant and functional in the community and who may potentially benefit from active urological interventions [10].

DOIC management is rarely straightforward. Each individu- al presents with a distinctive set of LUTS and functional impairment on filling and voiding. An underactive detrusor limits the use of anticholinergics, while options aiming to facilitate voiding can potentially worsen incontinence. The traditional approach to DOIC has focused on curtailing the DO symptoms with conservative means such as bladder retraining and fluid intake modification. Catheterization is reserved for those with high PVR volumes and risk of urinary retention. At our institution, treatment plans are formulated on the basis of principal symptomatic complaint, urodynamic findings, and patient tolerability. Medications are started at low doses and are titrated gradually according to patient response and side effects. Depending on flow and PVR volume, alpha-blockers and surgical options are frequently employed to maximize bladder emptying despite hypocontractility. Cautious doses of anticholinergics are given to those with bothersome storage symptoms. Some patients have received combination treatment (alpha-blockers and anticholinergic agents) aiming at both components of DOIC. Of the 48 men who were actively managed, 40 returned for follow-up: most (27) had responded positively and only 2 noted symptom deterioration. In the remaining 8 , it is plausible that some did not attend follow-up because of symptom resolution.

Only one case of urinary retention was seen during the follow-up period, and it was more likely to be secondary to disease progression than to be a treatment side effect (as this patient was taking alpha-blockers rather than anticholinergic agents). Our findings suggest that the presence of hypocontractility should not be an immediate contraindication to anticholinergic use. In fact, the efficacy of dose-titrated oxybutynin for geriatric incontinence was demonstrated previously in DOIC [23]. In our study, 21 patients in total were given anticholinergics alone or in combination with alpha-blockers; 13 improved on treatment, including 6 of 7 men with urge incontinence confirmed on urodynamics.

Interestingly, for those who returned for follow-up, 7 of 8 patients with features of both DO and reduced compliance on filling and 10 of 12 patients with evidence of abdominal straining on voiding improved on conventional pharmacotherapy (although our sample was too small to demonstrate statistical significance). This may appear counterintuitive at first, as those urodynamic findings seem to imply a higher degree of underlying detrusor dysfunction. The results suggest that the patients with more pronounced urodynamic findings may respond more readily to therapy.

Our study is limited by its retrospective design and the lack 
of validated questionnaires and repeat urodynamics used in the evaluation of treatment outcomes. However, given the current paucity of knowledge about the clinical course of patients with DOIC, we hope to shed some light on a clinical entity that has been regarded as difficult to manage.

In conclusion, the present study has demonstrated that conventional pharmacotherapy is reasonably effective for DOIC. In addition, the risk of developing acute urinary retention and sepsis is low with cautious dosing. The need for accurate diagnosis and characterization of voiding dysfunction using urodynamic studies in suitable elderly patients is emphasized.

\section{CONFLICT OF INTEREST}

No potential conflict of interest relevant to this article was reported.

\section{REFERENCES}

1. Resnick NM, Yalla SV. Detrusor hyperactivity with impaired contractile function. An unrecognized but common cause of incontinence in elderly patients. JAMA 1987;257:3076-81.

2. Yalla SV, Sullivan MP, Resnick NM. Update on detrusor hyperactivity with impaired contractility. Curr Bladder Dysfunct Rep 2007; 2:191-6.

3. Griffiths DJ, McCracken PN, Harrison GM, Gormley EA, Moore $\mathrm{KN}$. Urge incontinence and impaired detrusor contractility in the elderly. Neurourol Urodyn 2002;21:126-31.

4. Smith PP. Aging and the underactive detrusor: a failure of activity or activation? Neurourol Urodyn 2010;29:408-12.

5. Taylor JA 3rd, Kuchel GA. Detrusor underactivity: clinical features and pathogenesis of an underdiagnosed geriatric condition. J Am Geriatr Soc 2006;54:1920-32.

6. Yamamoto T, Sakakibara R, Uchiyama T, Liu Z, Ito T, Awa Y, et al. Neurological diseases that cause detrusor hyperactivity with impaired contractile function. Neurourol Urodyn 2006;25:356-60.

7. Kuo HC. Analysis of the pathophysiology of lower urinary tract symptoms in patients after prostatectomy. Urol Int 2002;68:99-104.

8. Kuo HC. Videourodynamic analysis of pathophysiology of men with both storage and voiding lower urinary tract symptoms. Urology 2007;70:272-6.

9. Abarbanel J, Marcus EL. Impaired detrusor contractility in com- munity-dwelling elderly presenting with lower urinary tract symptoms. Urology 2007;69:436-40.

10. Bromage SJ, Dorkin TJ, Chan L, Tse V. Urodynamics in the octogenarian female: is it worthwhile? Int Urogynecol J 2010;21:1117-21.

11. Resnick NM, Yalla SV. Geriatric incontinence and voiding dysfunction. In: Wein AJ, Kavoussi LR, Novick AC, Partin AW, Peters CA, editors. Campbell-Walsh urology. 9th ed. Philadelphia: Saunders; 2007. p. 2305-21.

12. Abrams P. Bladder outlet obstruction index, bladder contractility index and bladder voiding efficiency: three simple indices to define bladder voiding function. BJU Int 1999;84:14-5.

13. Katz GP, Blaivas JG. A diagnostic dilemma: when urodynamic findings differ from the clinical impression. J Urol 1983;129:1170-4.

14. Amundsen C, Lau M, English SF, McGuire EJ. Do urinary symptoms correlate with urodynamic findings? J Urol 1999;161:1871-4.

15. Lepor H, Rigaud G. The efficacy of transurethral resection of the prostate in men with moderate symptoms of prostatism. J Urol 1990;143:533-7.

16. Oesterling JE. Benign prostatic hyperplasia. Medical and minimally invasive treatment options. N Engl J Med 1995;332:99-109.

17. AUA Practice Guidelines Committee. AUA guideline on management of benign prostatic hyperplasia (2003). Chapter 1: Diagnosis and treatment recommendations. J Urol 2003;170(2 Pt 1):530-47.

18. Nitti VW, Kim Y, Combs AJ. Voiding dysfunction following transurethral resection of the prostate: symptoms and urodynamic findings. J Urol 1997;157:600-3.

19. Price DA, Ramsden PD, Stobbart D. The unstable bladder and prostatectomy. Br J Urol 1980;52:529-31.

20. Irani J, Brown CT, van der Meulen J, Emberton M. A review of guidelines on benign prostatic hyperplasia and lower urinary tract symptoms: are all guidelines the same? BJU Int 2003;92:937-42.

21. Aizenberg D, Sigler M, Weizman A, Barak Y. Anticholinergic burden and the risk of falls among elderly psychiatric inpatients: a 4-year case-control study. Int Psychogeriatr 2002;14:307-10.

22. Berdot S, Bertrand M, Dartigues JF, Fourrier A, Tavernier B, Ritchie $\mathrm{K}$, et al. Inappropriate medication use and risk of falls: a prospective study in a large community-dwelling elderly cohort. BMC Geriatr 2009;9:30.

23. Miller KL, DuBeau CE, Bergmann MA, Juliano KL, Resnick NM. Dose titration key to oxybutynin efficacy for geriatric incontinence, even for DHIC [abstract]. Neurourol Urodyn 2000;19:538-9. 